\title{
Establishment of an induced memory response in Pseudomonas aeruginosa during infection of a eukaryotic host
}

\author{
Adrian Kordes $\mathbb{B}^{1} \cdot$ Nora Grahl $^{1} \cdot$ Michal Koska ${ }^{1} \cdot$ Matthias Preusse $\mathbb{D}^{2} \cdot$ Alejandro Arce-Rodriguez ${ }^{2}$. \\ Wolf-Rainer Abraham $\mathbb{1}^{3} \cdot$ Volkhard Kaever $^{4} \cdot$ Susanne Häussler ${ }^{1,2}$
}

Received: 19 November 2018 / Revised: 14 March 2019 / Accepted: 15 March 2019 / Published online: 5 April 2019

(c) International Society for Microbial Ecology 2019

\begin{abstract}
In a given habitat, bacterial cells often experience recurrent exposures to the same environmental stimulus. The ability to memorize the past event and to adjust current behaviors can lead to efficient adaptation to the recurring stimulus. Here we demonstrate that the versatile bacterium Pseudomonas aeruginosa adopts a virulence phenotype after serial passage in the invertebrate model host Galleria mellonella. The virulence phenotype was not linked to the acquisition of genetic variations and was sustained for several generations, despite cultivation of the ex vivo virulence-adapted $P$. aeruginosa cells under rich medium conditions in vitro. Transcriptional reprogramming seemed to be induced by a host-specific food source, as reprogramming was also observed upon cultivation of $P$. aeruginosa in rich medium supplemented with polyunsaturated long-chain fatty acids. The establishment of induced memory responses adds a time dimension and seems to fill the gap between long-term evolutionary genotypic adaptation and short-term induced individual responses. Efforts to unravel the fundamental mechanisms that underlie the carry-over effect to induce such memory responses will continue to be of importance as hysteretic behavior can serve survival of bacterial populations in changing and challenging habitats.
\end{abstract}

\section{Introduction}

Pseudomonas aeruginosa is a versatile environmental bacterium and opportunistic human pathogen, which can thrive in a large variety of different and challenging habitats. The natural environment can serve as a reservoir

Supplementary information The online version of this article (https:// doi.org/10.1038/s41396-019-0412-1) contains Supplementary

Material, which is available to authorized users.

$\triangle$ Susanne Häussler

Susanne.Haeussler@helmholtz-hzi.de

1 Department of Molecular Bacteriology, TWINCORE Centre for Experimental and Clinical Infection Research, Hannover 30625, Germany

2 Department of Molecular Bacteriology, Helmholtz Centre for Infection Research, Braunschweig 38124, Germany

3 Department of Chemical Microbiology, Helmholtz Centre for Infection Research, Braunschweig 38124, Germany

4 Research Core Unit Metabolomics and Institute of Pharmacology, Hannover Medical School, Hannover 30625, Germany for $P$. aeruginosa to occasionally cause opportunistic infections in a wide range of hosts and tissues [1,2]. Most of those host habitats are sub-optimal for sustained growth and thus provide strong selection for adaptive changes. Especially in the chronically infected cystic fibrosis lung, $P$. aeruginosa populations exist for a long-enough time for the positive selection of adaptive mutations to take place $[3,4]$. Several studies have shown that there are a number of bacterial traits that show the signature of within-patient directional selection [5-7].

In addition to the evolution of adaptive mutations, phenotypic plasticity allows bacterial adaptation to a changing environment [8-11]. Although genetic diversity increases the potential of whole populations to adapt to changes in environmental conditions, a plastic phenotypic response to variations in the environment increases the fitness of the individual bacterium [12]. More recently, it has been argued that another response mechanism fills the gap between long-term evolutionary adaptation and short-term individual response [13]. This includes the incorporation of a time dimension and is characterized by the expression of a phenotypic response for several generations despite a change in the 
conditions that first induced the phenotypic response. This hysteretic behavior is also called response memory, as gene expression persists after removal of the external inducer [12, 14-16].

In this study we exposed a clinical $P$. aeruginosa isolate to the challenging environment within a eukaryotic host. We aimed to evaluate whether virulence could be induced upon host passages and which factors would be involved in the expression of virulence. Infections of caterpillar larvae have increasingly been used as a surrogate to study host-pathogen interactions in a range of microorganisms including $P$. aeruginosa [17-22]. We therefore passaged the $P$. aeruginosa clinical isolate multiple times in larvae of the wax moth Galleria mellonella. The immune system of the G. mellonella larvae shows functional similarities to human innate immune responses such as expression of antimicrobial peptides, lysozyme, generation of reactive oxygen species, and phagocytosis of invading microbes [23-26].

Genotypic and phenotypic analyses of the in vivopassaged $P$. aeruginosa isolate uncovered extensive transcriptional reprogramming and phenotypic switching to higher virulence. This phenotypic switch could be in large parts recapitulated by growing the bacteria for several passages in rich medium with an excess of long-chain polyunsaturated fatty acids. The latter have been demonstrated to be enriched in the hemolymph of the insect larvae [27]. Importantly, the phenotypic changes were not associated with genotypic changes and were lost after cultivation of the in vivo and the in vitro evolved strain for several passages under rich medium culture conditions. Our results highlight that in addition to a non-inducible genotypic and a wellstudied inducible plastic response, the opportunistic pathogen $P$. aeruginosa also produces memory phenotypes. These seem to be induced by a host-specific food source and persist for several generations. The establishment of induced memory responses might confer an advantage in bacteria that live in fluctuating and challenging environments.

\section{Materials and methods}

\section{Strains used in this study}

The 414 P. aeruginosa isolates (Fig. 1a) that were included in this study were collected at five major sampling sites from clinical microbiology laboratories of hospitals across Germany and at sampling sites in Spain, Hungary, and Romania. In addition, $P$. aeruginosa isolates were collected in German private practice laboratories or provided by strain collection curators [28]. For all of these isolates, transcriptional profiles were recorded in vitro under one standard environmental condition (lysogeny broth (LB) medium $\mathrm{OD}_{600}=2$ ) with the aim to correlate gene expression to virulence (this study) as well as to antibiotic resistance phenotypes (to be published elsewhere). Isolate CH2658 was chosen for in vivo and in vitro evolution experiments in this study, because this isolate exhibited a low virulence phenotype but still survived the passages in the larvae. This isolate was obtained from the lab of P. Gastmeier, Charite Berlin, Germany. The in vivo-passaged strains CH2658 I-IV correspond to passages $1-4$. The last passage CH2658 IV is referred to as the "evolved strain." The evolved strain was passaged in LB (4 days, two passages a day) to generate the revertants $\mathrm{CH} 2658$ Rev1-4 corresponding to samples from day 1 to 4 . The last passage $\mathrm{CH} 2658$ Rev4 is referred to as "revertant". In addition, the clinical isolate was passaged under in vitro conditions in the presence of linolenic acid (Roth) with $(\mathrm{CH} 2658 \mathrm{Lil}+\mathrm{P})$ and without paraffin $(\mathrm{CH} 2658 \mathrm{Lil})$ in duplicates. As controls, $\mathrm{CH} 2658$ was passaged in $\mathrm{LB}(\mathrm{CH} 2658 \mathrm{LB})$ and in $\mathrm{LB}$ supplemented with paraffin $(\mathrm{CH} 2658 \mathrm{LB}+\mathrm{P})$. The in vitro passage experiment was conducted for 4days and two passages a day (in duplicates).

\section{G. mellonella infection and passaging experiments}

Based on visual inspection, healthy G. mellonella larvae (faunatopics, Germany) were selected for the infection experiments. To prepare the inoculum, bacteria were grown overnight, washed in sterile phosphate-buffered saline (PBS), and adjusted to a final concentration of 5000 cells $/ \mathrm{ml}$ by serial dilution in PBS. For each individual experiment, ten larvae were infected in parallel by injecting $20 \mu \mathrm{l}$ of the $P$. aeruginosa inoculum ( $\sim 100$ colony-forming units) into the last left proleg using a $500 \mu \mathrm{l}$ Hamilton syringe and a 30 $\mathrm{G}$ needle. PBS was injected as a control. The larvae were incubated at $37^{\circ} \mathrm{C}$. Death was defined by two major criteria, which were assessed by visual inspection at 24,40 , and $48 \mathrm{~h}$ following infection: loss of movement in response to stimulation and melanization of the cuticle.

For in vivo passaging, a multiplicity of infection (MOI) of 1000 cells was used (Fig. 1b). After $24 \mathrm{~h}$ of incubation of the infected larvae at $37^{\circ} \mathrm{C}$, the hemolymph was collected via puncturing the larvae. The hemolymph was pooled from ten larvae, diluted ten times in PBS, and plated on cetrimid agar to select for $P$. aeruginosa bacteria. Bacterial colonies were washed off the agar for the next infection round (1000 cell bottleneck). In total, four infection rounds were conducted. Glycerol stocks from every passage were frozen at $-80^{\circ} \mathrm{C}$. The evolved strain (CH2658 IV) was used to generate revertants (Fig. 1b). Therefore, single colonies of the evolved strain were passaged in LB medium with shaking (180 r.p.m.) at $37^{\circ} \mathrm{C}$ in four independent experiments. An inoculum of $\mathrm{OD}_{600}=0.03$ of the $\mathrm{LB}$ culture was transferred every $12 \mathrm{~h}$ for a total of 4 days. The clinical 
A

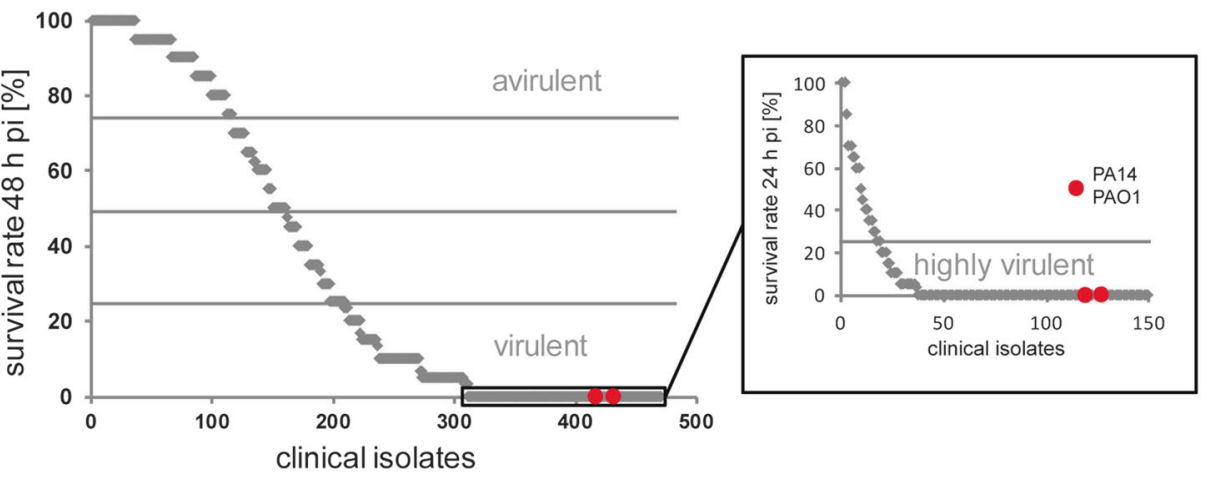

B

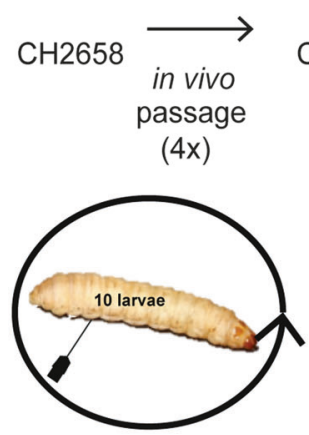

$\mathrm{CH} 2658$

Rev

C

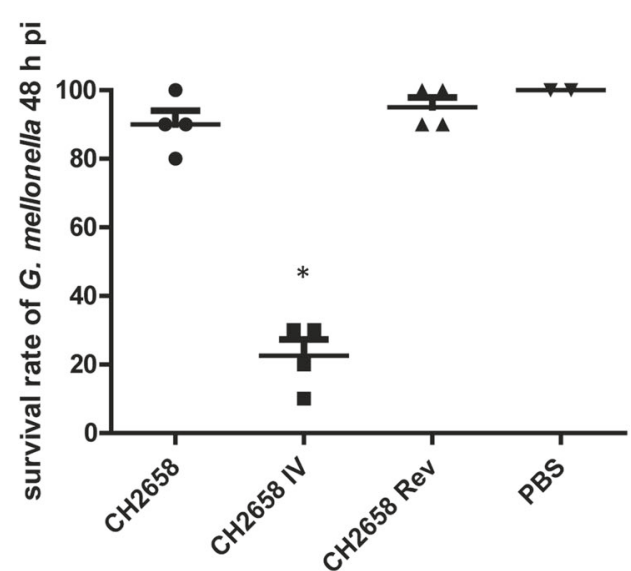

Fig. 1 G. mellonella as a model system to study $P$. aeruginosa virulence. a \% Survival values (mean of biological replicates) of larvae infected with overall 414 clinical $P$. aeruginosa isolates were recorded $48 \mathrm{~h}$ post infection (pi) (left). For the most virulent isolates, also survival rates $24 \mathrm{~h}$ pi were determined (right). The P. aeruginosa reference strains, PA14 and PAO1, are highlighted in red. b The less virulent clinical isolate $\mathrm{CH} 2658$ was passaged four times in the G. mellonella larvae to generate the evolved strain $\mathrm{CH} 2658$ IV. The evolved strain $\mathrm{CH} 2658$ IV was passaged (eight times) in rich LB medium for 4 days to generate the revertant $\mathrm{CH} 2658$ Rev. c The survival rates of $G$. mellonella larvae infected with the clinical isolate (CH2658), the evolved strain (CH2658 IV), and the revertant (CH2658 Rev) were recorded in four biological replicates. PBS was injected as a control $(n=2)$. Asterisks indicate parameters that show statistically significant differences $(p<0.05)$ as determined by the Mann-Whitney $U$-test isolate was also passaged in LB medium supplemented with $5 \%$ of linolenic acid with and without the addition of paraffin. Paraffin was added in order to generate a microaerophilic environment. Using an inoculation of $\mathrm{OD}_{600}=$ 0.05 , the bacterial culture was passaged eight times every $12 \mathrm{~h}$ in fresh media. The experiment was performed in duplicates (Fig. 6).

\section{DNA and RNA sequencing}

For RNA extraction, bacteria were grown in $10 \mathrm{ml} \mathrm{LB}$ to early stationary phase $\left(\mathrm{OD}_{600}=2\right)$. The RNeasy Mini Kit (Qiagen) and Qiashredder columns (Qiagen) were used according to the manufacturer's instruction. Obtained RNA was quality checked using the RNA Nano Kit on an Agilent Bioanalyzer 2100 (Agilent Technologies). Ribosomal RNA was removed using the Ribo-Zero Bacteria Kit (Illumina) and cDNA libraries were generated with the ScriptSeq v2 Kit (Illumina). The samples were sequenced in single-end mode on an Illumina HiSeq 2500 device $(1 \times 50$ bp reads $)$. Mapping was performed using a stampy pipeline [29] with the PA14 genome as a reference. The RNA sequencing (RNA-Seq) data of the clinical isolate, all passages, and revertants used in this study were uploaded to NCBI's Gene Expression Omnibus (GSE122938).

The RNA-Seq data from the 414 clinical isolates were uploaded to NCBI's Gene Expression Omnibus (GSE123544). Differential gene expression and principal component analysis (PCA) were performed using the $\mathrm{R}$ package DESeq2 (v.1.18.1) (Fig. 5, Supplementary Fig. S7, and Supplementary Table S1) [30]. Normalized reads per gene (nrpg) were calculated by the default settings of the $\mathrm{R}$ package DESeq2. Area proportional Venn diagrams were generated using the online tool BioVenn (Fig. 6) [31].

DNA was extracted from cell pellets derived from overnight cultures using the DNeasy Blood \& Tissue Kit (Qiagen) and fragmented with the S2/E210 Focused-ultrasonicator (Covaris) to achieve $300 \mathrm{nt}$-long fragments. Library 
preparation was performed using NEBNext Ultra DNA Library Prep Kit for Illumina (NEB) according to the manufacturer's instructions. The samples were sequenced in pairedend mode $(2 \times 250 \mathrm{bp}$ reads $)$ on an Illumina HiSeq 2500 device. Clipped reads were mapped to the PA14 reference genome as described for RNA-Seq mapping. All DNA-seq data were uploaded to SRA database under the reference number PRJNA506546. For single-nucleotide polymorphism (SNP) detection, samtools (v.0.1.19) and parSNP (v.1.2) were used with PA14 genome as a reference. After quality filtering, every identified SNP position was re-checked manually in the integrative genome viewer (IGV) (v.2.3.98).

\section{Correlation of virulence phenotypes and gene expression data of 414 clinical isolates}

We calculated the differential gene expression between the most virulent and the less virulent clinical isolates using edgeR (v.3.20.1) (Supplementary Table S2) [32]. Therefore, the transcription profiles that were recorded under rich medium conditions of the virulent isolates $(0 \%$ survival $24 \mathrm{~h}$ post infection $=110$ isolates) and less virulent isolates $(100 \%$ survival $48 \mathrm{~h}$ post infection $=31$ isolates $)$ ) were treated as replicates, respectively. We also selected groups of clinical isolates, which expressed each individual gene at high and low levels. Therefore, the expression values (nrpg) for each gene (overall 5946 genes) across all clinical isolates were determined and the group of 50 isolates showing the highest and the group of 50 isolates exhibiting the lowest expression of all individual genes were defined (Fig. 3a, upper panel). Next, we assigned the data of the killing efficiency as determined in the G. mellonella assay to the 50 high and the 50 low gene expression clinical isolates across all individual genes (Fig. 3a, lower panel). The Wilcoxon-Mann-Whitney $U$-test was applied to identify significant associations between gene expression values and virulence phenotypes (Fig. 3b).

\section{Determination of the generation times required for phenotype reversion}

In four independent experiments, 25000 cells of $\mathrm{CH} 2658 \mathrm{IV}$ were used to inoculate $10 \mathrm{ml}$ of LB medium. Cultures were grown at $37^{\circ} \mathrm{C}$ and every $12 \mathrm{~h} 25000$ cells were transferred into fresh LB medium. The generation time over 3 days in overall six passages was calculated by subtracting the $\log 2$ cell number at the start point by the $\log 2$ cell number after $12 \mathrm{~h}$. We found a mean duplication time of $45 \mathrm{~min}$ (Supplementary Fig. S3). Every $12 \mathrm{~h}$, we also plated an aliquot of the cultures on blood agar plates and monitored the colony morphologies. The evolved strain exhibited a smaller colony size and could be distinguished from the original clinical isolate by visual inspection (Supplementary Fig. S4).

\section{Cytotoxicity in A549 cells}

A549 cells were seeded into 24 -well plates at a density of $5 \times$ $10^{4}$ cells/well and incubated in Dulbecco's modified Eagle's medium (DMEM) complete (DMEM high glucose (ThermoFisher) supplemented with $2 \mathrm{mM} \mathrm{L}$-glutamine (ThermoFisher), $1 \times$ non-essential amino acids (ThermoFisher), $10 \%$ fetal bovine serum (Sigma-Aldrich), $100 \mathrm{U} / \mathrm{ml}$ PenicillinStreptomycin (ThermoFisher)) at $37{ }^{\circ} \mathrm{C}$ and $5 \% \mathrm{CO}_{2}$ until $90 \%$ confluency. The actual cell number per well was determined before infection. $P$. aeruginosa cells were grown to early stationary phase and washed in DMEM complete without phenol red and antibiotics. Bacterial cells were adjusted to an MOI of 10 before they were added to the PBSwashed A549 cells followed by a brief centrifugation ( $5 \mathrm{~min}$ at $500 \times g$ ). After 3 and $22 \mathrm{~h}$ of incubation, the supernatants were collected, and bacterial cells and cell debris were removed by centrifugation. As a positive control, a cytomax sample was treated with lysis buffer $45 \mathrm{~min}$ before the collection of supernatant and cytotoxicity of all samples was determined via lactate dehydrogenase (LDH) assay following the manufacturer's instructions (PierceTM LDH Cytotoxicity Assay Kit; ThermoFisher) (Supplementary Fig. S2).

\section{Biofilm measurement with crystal violet}

The crystal violet assay was performed as described previously [33]. Briefly, overnight-grown, PBS-washed bacteria were inoculated in $100 \mu \mathrm{l}$ of $\mathrm{LB}$ (at an $\mathrm{OD}_{600}$ of 0.02) in 96-well microtiter plates (flexible plate, PVC, U bottom, BD Biosciences). The plates were incubated with an airpermeable membrane at $37^{\circ} \mathrm{C}$ at a humid atmosphere for $24 \mathrm{~h}$. The wells were washed twice with $200 \mu \mathrm{l}$ distilled $\mathrm{H}_{2} \mathrm{O}$ to remove the media and unattached cells. One hundred and fifty microliters of crystal violet staining solution $\left(0.1 \% \mathrm{~m} / \mathrm{v}\right.$ in $\left.\mathrm{H}_{2} \mathrm{O}\right)$ was added and incubated for $30 \mathrm{~min}$ at room temperature. Wells were washed twice and dried for $30 \mathrm{~min}$ at room temperature. To de-stain the biofilm, $200 \mu \mathrm{l}$ ethanol (95\%) was added and incubated for $30 \mathrm{~min}$. One hundred and twenty-five microliters of the ethanol solution was transferred into a fresh 96-well plate (hart plastic, flat bottom) and absorbance at $550 \mathrm{~nm}$ was measured (Figs. 4b and $6 c$, and Supplementary Fig. S6).

\section{Quantification of cyclic-di-GMP levels}

Extraction and quantification of cyclic-di-GMP (c-diGMP) by the use of high-performance liquid chromatography-coupled tandem mass spectrometry was performed as described previously with some modifications $[34,35]$. Briefly, $5 \mathrm{ml}$ of a bacterial suspension were collected after $24 \mathrm{~h}$ of cultivation in LB at $37^{\circ} \mathrm{C}$. Before extraction [methanol:acetonitrile:water; 2:2:1], isotope- 
labeled $13 \mathrm{C} 15 \mathrm{~N}-\mathrm{c}-\mathrm{di}-\mathrm{GMP}$ was added as an internal standard. C-di-GMP extracts were stored overnight at $-20{ }^{\circ} \mathrm{C}$ and c-di-GMP concentrations (pmol c-di-GMP/ $\mathrm{mg}$ protein) were measured in three independent experiments, each analyzed in duplicates (Figs. 4c and $6 d$ ). The protein concentration was determined by the use of the Roti Nanoquant solution for Bradford protein assays according to the manufacturer's instructions (Roth).

\section{Motility}

Motility assays were performed as previously described [36]. Swimming assays were performed in BM2 medium with $0.3 \%$ agar and swarming assays in BM2 medium with $0.5 \%$ agar. Plates were incubated at $30^{\circ} \mathrm{C}$ for $16 \mathrm{~h}$ before optical assessment of swimming and swarming activity from two biological replicates (Fig. 4a).

\section{Fatty acid measurement}

To determine the composition of fatty acids in $P$. aeruginosa, the cell pellets (grown to an $\mathrm{OD}_{600}=2$ ) were saponified [MeOH:NaOH $(15 \%) 1: 1,1 \mathrm{~h}, 100^{\circ} \mathrm{C}$ ], derivatized

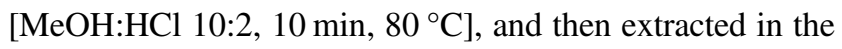
organic phase as described previously [37]. Samples were analyzed by gas chromatography on an Agilent 6890 N GC with FID (flame ionization detector). Separation of the fatty acids methyl esters were carried out with a Macherey Nagel Optima 5 column (5\% phenyl, $95 \%$ dimethylpolysiloxane; $50 \mathrm{~m}$ length; $0.32 \mathrm{~mm}$ inner diameter; $0.25 \mu \mathrm{m}$ film thickness). Individual fatty acid methyl esters were identified by their retention time compared with standards and quantified as percentages $(\%)$ of the total amount of fatty acids per sample (Fig. 4d).

\section{Results}

\section{$P$. aeruginosa clinical isolates exhibit a broad distribution of virulence phenotypes in the $G$. mellonella infection model}

In this study, we used the previously established G. mellonella infection model [38] to screen overall 414 clinical $P$. aeruginosa strains for their virulence phenotype. As depicted in Fig. 1a, a broad distribution of virulence phenotypes was observed. There were many virulent isolates that killed $100 \%$ of the larvae within $48 \mathrm{~h}$ and also a proportion of highly virulent isolates that killed $100 \%$ of larvae within $24 \mathrm{~h}$ post infection. Furthermore, almost less virulent isolates were found as well as virulent phenotypes at all intermediate levels. Interestingly, the two $P$. aeruginosa reference strains PA14 and PAO1 exhibited a highly virulent phenotype. Both killed the larvae within $24 \mathrm{~h}$.

\section{Virulence can be increased in a low virulent clinical $P$. aeruginosa isolate upon serial passages in the $G$. mellonella infection model}

With the aim to study bacterial adaptation during infection of the G. mellonella larvae and to evaluate whether virulence can be induced upon infection, we selected a low virulent $P$. aeruginosa clinical isolate $(\mathrm{CH} 2658)$ and passaged it overall four times in the G. mellonella infection model (Fig. 1b). We observed that virulence increased in the passaged strain (CH2658 IV) and reached the virulence potential of the virulent clinical isolates (Fig. 1c and Supplementary Fig. S1). During the passages, killing of the larvae became faster. This could mean that higher cell numbers are reached in shorter time periods, because the bacteria grow faster. Nevertheless, in accordance with an increased production of virulence factors, the evolved strain (CH2658 IV) also exhibited a significantly increased cytotoxicity in A549 lung epithelial cells as compared with its ancestor (Supplementary Fig. S2A), indicating that the expression of virulence factors could also provide the bacteria with additional nutrients, so that they grow faster.

\section{Transcriptional profile of the evolved $P$. aeruginosa isolate}

To obtain more insights into the gained virulence traits, we performed RNA-Seq of the clinical isolate (CH2658) and the evolved strain (CH2658 IV). We found an upregulation of the PseudoCAP categories protein secretion/export apparatus, motility and attachment, and secreted factors (Fig. 2). More specifically, we found higher expression of genes involved in type III secretion (T3SS) in the evolved strain and an enrichment of pili gene expression (a complete list of differentially expressed genes can be found in Supplementary Table S1). Furthermore, genes involved in glycerol metabolism and uptake were upregulated. Overall, less genes (120) were upregulated and more genes (463) were downregulated in the evolved strain ( $\log 2$ fold change $\geq 1.5, p$-value $<0.05$, Supplementary Table $\mathrm{S} 1)$. Among the downregulated genes we found, e.g., genes involved in the production of extracellular matrix (pslO, $p s l N, p s l J, a l g L$, algG, algI, alg8) or glycogen metabolism ( $g \lg A, g \lg B, g \lg P$, $g \lg X$ ). In addition, genes involved in general metabolism were downregulated in the $\mathrm{CH} 2658$ IV strain, e.g., genes involved in carbon compound catabolism, chemotaxis, adaption, and protection or energy metabolism (Supplementary Table S1 and Fig. 2). 


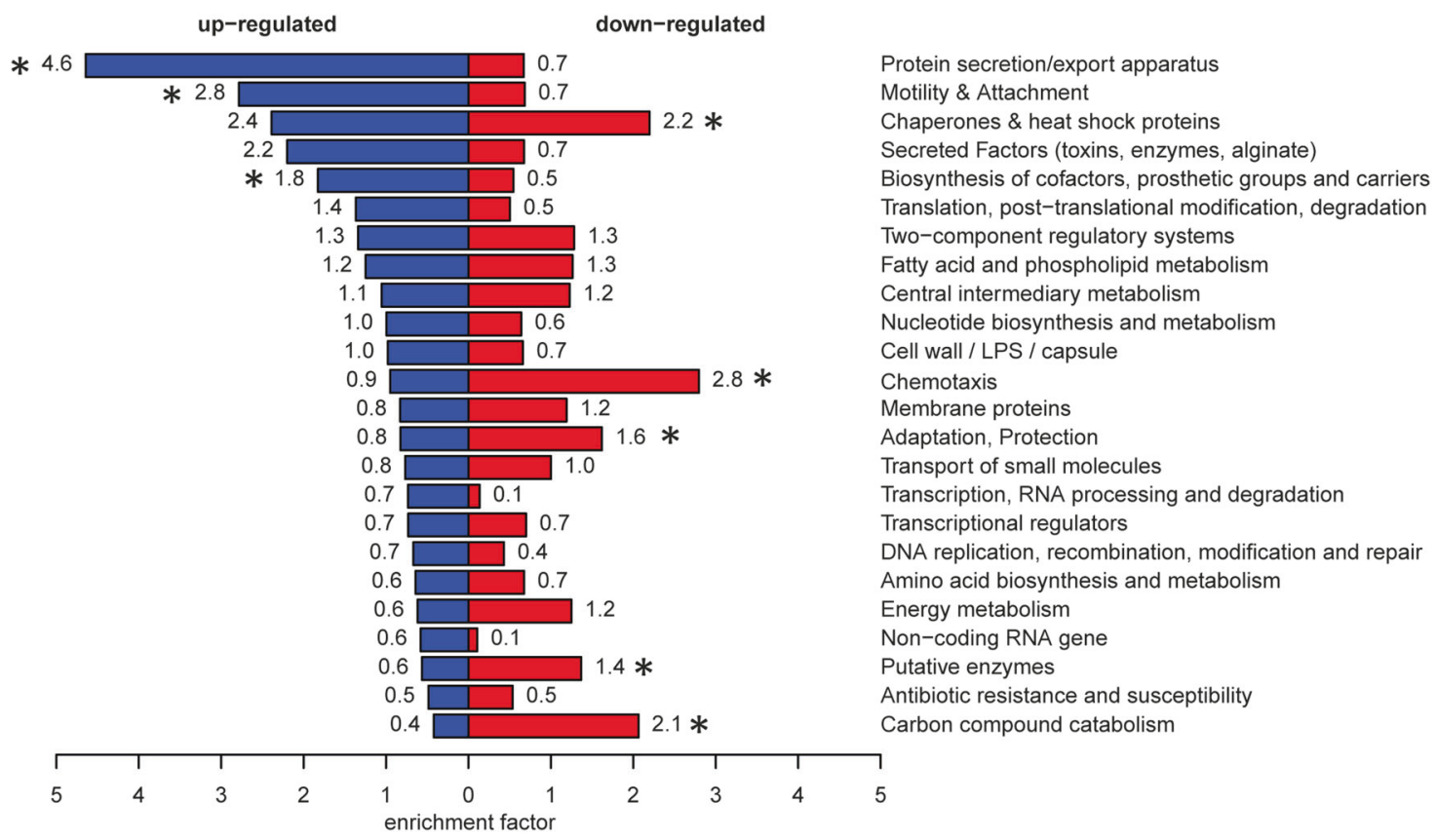

Fig. 2 Transcriptional analysis uncovers phenotypic switching of CH2658 IV. Genes that were differentially expressed under rich medium conditions ( $\log 2$ fold change $\geq 1.5$ ) between the evolved $\mathrm{CH} 2658$ IV strain and the ancestor clinical isolate $\mathrm{CH} 2658$ were assigned to PseudoCAP categories (a complete list of the differentially expressed genes can be found in Supplementary Table S1). The ratio of the abundance of differentially regulated genes (blue, upregulated;

\section{The evolved $P$. aeruginosa isolate adopts expression of genes that can be linked to a virulence phenotype in the $G$. mellonella model}

We next were interested to evaluate the contribution of the transcriptionally reprogrammed genes to the virulence phenotype in the insect larvae. For all 414 clinical strains, which have been tested in this study for their virulence phenotype in the G. mellonella model (Fig. 1), we have also generated in vitro transcriptional profiles recorded under rich medium conditions. Here we selected from the group of 414 clinical isolates the 110 most virulent and the 31 least virulent strains (see Material and Methods section for the selection criteria of the clinical isolates) and determined the differentially expressed genes between these two groups. We could show that in accordance with the gene expression profile of the in vivo evolved isolate, the highly virulent clinical isolates upregulate T3SS-related genes, whereas genes important for alginate production are downregulated (alginate operon PA14_18470-18565, algA, algB, algD, alg $F$, algI, algJ; Supplementary Table S2).

Next, we aimed at elucidating which single genes have the highest impact on the bacterial virulence phenotype. Figure 3 depicts the contribution of the expression of 5946 P. aeruginosa genes to the virulence phenotype in the G. mellonella model. Therefore, clinical isolates that express the individual red, downregulated) within the distinct PseudoCAP categories to the abundance of genes in the respective category was determined, as well as the ratio of the abundance of genes within the respective category to the total number of expressed genes. The enrichment factor depicted here represents the ratio of these two values. Asterisks indicate parameters that show statistically significant differences $(p<0.05)$ as determined by hypergeometrical distribution

$P$. aeruginosa genes at high vs. low levels were identified and the difference in the virulence potential between the two groups of clinical isolates was assessed. The expression of 389 genes could be positively associated with bacterial virulence in the G. mellonella assay, whereas the expression of 534 genes was negatively associated with bacterial virulence (Supplementary Table S4). Figure 3b highlights those genes that were found in this study to be differentially regulated in the evolved strain. Clearly, we found many genes that were shown to be upregulated in the evolved strain, and that were also highly expressed in virulent clinical isolates as compared with the less virulent ones, e.g., the expression patterns of T3SS-related genes and pil genes were significantly correlated to the virulence phenotype (Fig. 3c). Vice versa, we also found genes such as the alg genes, which were downregulated in the evolved strain as well as in the clinical isolates that exhibited a high virulence phenotype.

\section{Phenotypic change was not linked to genetic variation}

In order to identify genomic mutations that might underlie the establishment of the more virulent phenotype within the in vivo evolved strain, we extracted DNA from every in vivo passage $(\mathrm{CH} 2658 \mathrm{I}-\mathrm{IV})$ and sequenced the DNA. The genomes were sequenced at overall $88 \times$ coverage 

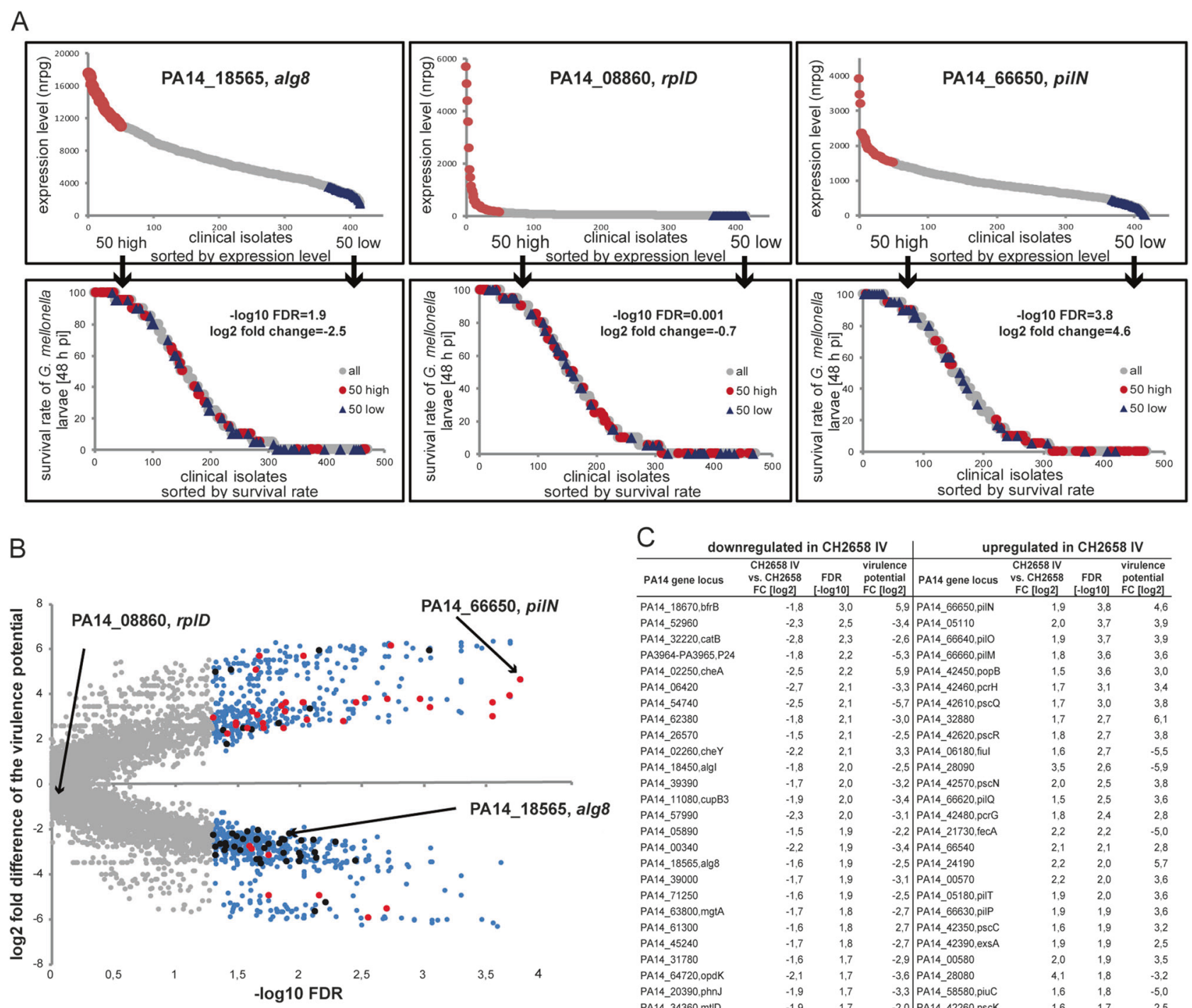

\begin{tabular}{|c|c|c|c|c|c|c|c|}
\hline \multicolumn{4}{|c|}{ downregulated in $\mathrm{CH} 2658$ IV } & \multicolumn{4}{|c|}{ upregulated in $\mathrm{CH} 2658 \mathrm{IV}$} \\
\hline PA14 gene locus & $\begin{array}{l}\text { CH2658 IV } \\
\text { vs. CH2658 } \\
\text { FC [log2] }\end{array}$ & $\begin{array}{c}\text { FDR } \\
{[-\log 10]}\end{array}$ & $\begin{array}{l}\text { virulence } \\
\text { potential } \\
\text { FC [ }[\log 2]\end{array}$ & PA14 gene locus & $\begin{array}{l}\text { CH2658 IV } \\
\text { vs. CH2658 } \\
\text { FC [log2] }\end{array}$ & $\begin{array}{c}F D R \\
{[-\log 10]}\end{array}$ & $\begin{array}{l}\text { virulence } \\
\text { potential } \\
\text { FC [ }[\log 2]\end{array}$ \\
\hline PA14_18670,bfrB & $-1,8$ & 3,0 & 5,9 & PA14_66650,pilN & $\begin{array}{r}1,9 \\
\end{array}$ & 3,8 & 4,6 \\
\hline PA14_52960 & $-2,3$ & 2,5 & $-3,4$ & PA14_05110 & 20 & 3,7 & 3,9 \\
\hline PA14_32220,catB & $-2,8$ & 2,3 & $-2,6$ & PA14_66640,pilO & 1,9 & 3,7 & 3,9 \\
\hline PA3964-PA3965.P24 & $-1,8$ & 2.2 & $-5,3$ & PA14_66660.pilM & 1,8 & 3.6 & 3.6 \\
\hline PA14_02250,cheA & $-2,5$ & 2,2 & 5,9 & PA14_42450,popB & 1,5 & 3,6 & 3,0 \\
\hline PA14_06420 & $-2,7$ & 2,1 & $-3,3$ & $\mathrm{PA}^{-} 4-42460, \mathrm{pcrH}$ & 1,7 & 3,1 & 3,4 \\
\hline PA14_54740 & $-2,5$ & 2,1 & $-5,7$ & PA14_42610,pscQ & 1,7 & 3,0 & 3,8 \\
\hline PA14_62380 & $-1,8$ & 2,1 & -3.0 & PA14_32880 & 1.7 & 2,7 & 6.1 \\
\hline PA14_26570 & $-1,5$ & 2,1 & $-2,5$ & PA14_42620,pscR & 1,8 & 2,7 & 3,8 \\
\hline PA14_02260,cheY & $-2,2$ & 2,1 & 3,3 & PA14_06180,fiul & 1,6 & 2,7 & $-5,5$ \\
\hline PA14_18450,algl & $-1,8$ & 2,0 & $-2,5$ & PA14_28090 & 3,5 & 2,6 & $-5,9$ \\
\hline PA14_39390 & $-1,7$ & 2,0 & -3.2 & PA14_42570.pscN & 2,0 & 2,5 & 3.8 \\
\hline PA14_11080,cupB3 & $-1,9$ & 2,0 & $-3,4$ & PA14_66620,pilQ & 1,5 & 2,5 & 3,6 \\
\hline PA14_57990 & $-2,3$ & 2,0 & $-3,1$ & PA14_42480.pcrG & 1,8 & 2,4 & 2,8 \\
\hline PA14_05890 & $-1,5$ & 1,9 & $-2,2$ & PA14_21730,fecA & 2,2 & 2,2 & $-5,0$ \\
\hline PA14_00340 & $-2,2$ & 1,9 & $-3,4$ & PA14_66540 & 2.1 & 2.1 & 2.8 \\
\hline PA14_18565,alg8 & $-1,6$ & 1,9 & $-2,5$ & PA14_24190 & 2,2 & 2,0 & 5,7 \\
\hline PA14_39000 & $-1,7$ & 1,9 & $-3,1$ & PA14_00570 & 2.2 & 2,0 & 3.6 \\
\hline PA14_71250 & $-1,6$ & 1,9 & $-2,5$ & PA14_05180,pilT & 1,9 & 2,0 & 3,6 \\
\hline PA14_63800,mgtA & $-1,7$ & 1.8 & $-2,7$ & PA14_66630,pilP & 1.9 & 1.9 & 3.6 \\
\hline PA14_61300 & $-1,6$ & 1,8 & 2,7 & PA14_42350,pscC & 1,6 & 1,9 & 3,2 \\
\hline PA14_45240 & $-1,7$ & 1,8 & $-2,7$ & PA14_42390,exsA & 1,9 & 1,9 & 2.5 \\
\hline PA14_31780 & $-1,6$ & 1,7 & $-2,9$ & PA14_00580 & 2,0 & 1,9 & 3,5 \\
\hline PA14_64720,opdK & $-2,1$ & 1,7 & $-3,6$ & PA14_28080 & 4.1 & 1,8 & $-3,2$ \\
\hline PA14_20390,phnJ & $-1,9$ & 1,7 & $-3,3$ & PA14_58580,piuc & 1,6 & 1,8 & -5.0 \\
\hline PA14_34360,mtID & $-1,9$ & 1,7 & $-2,0$ & PA14_42260,psck & 1.6 & 1,7 & 2.5 \\
\hline PA14_02640,mcdM & $-1,9$ & 1,7 & $-2,5$ & PA14_14330 & 2,5 & 1,7 & 2,7 \\
\hline PA14_64890 & $-2,8$ & 1.7 & $-2,5$ & PA14_62260 & 2.4 & 1.7 & 5,7 \\
\hline PA14_31990,czcB & $-3,0$ & 1,7 & $-3,3$ & PA14_42600,pscP & 1,5 & 1,7 & 3,1 \\
\hline PA14_18470,algL & $-2,0$ & 1.7 & $-2,5$ & PA14_51530,exoU & 3,9 & 1,7 & 5,1 \\
\hline
\end{tabular}

Fig. 3 Correlation of virulence phenotypes and gene expression data of 414 clinical isolates. a The 414 clinical isolates were ranked according to the expression levels of single genes (normalized reads per gene, total of 5946 PA14 genes) as exemplarily shown for three genes (alg8, $r p l D, \operatorname{pil} N)$ (a, upper row). The 50 isolates that exhibited the highest and lowest expression values of each single gene (indicated in red and blue, respectively) were chosen and their virulence phenotype (survival rate of $G$. mellonella larvae $48 \mathrm{~h}$ pi) was assigned (a, lower row). The fold change in the difference of the virulence potential between the groups of high vs. low gene expression was determined (Wilcoxon-Mann-Whitney $U$-test with corrected $p$-values (FDR)). b Volcano plot showing the fold difference $(y$-axis) and the

(mean value). ParSNP-based SNP calling revealed in total six SNPs between the clinical isolate $\mathrm{CH} 2658$ and the evolved strain $\mathrm{CH} 2658$ IV. However, manual re-checking in the IGV revealed that all of them were located in regions of low genome coverage and thus were defined as false positive. Thus, genetic variations do not seem to be the reason for the phenotypic change. corresponding $p$-values ( $x$ axis) in the virulence potential of the groups of 50 clinical isolates expressing the individual $P$. aeruginosa genes at high vs. low levels (each dot corresponds to one individual P. aeruginosa gene). Significant differences in virulence potentials are depicted in blue. Genes that were also found to be differentially regulated (fold change $\geq 1.5$ ) in the $G$. mellonella evolved strain vs. the ancestor isolate $\mathrm{CH} 2658$ are highlighted in red (upregulated in the evolved isolate) and black (downregulated in the evolved isolate). c The most significant genes that are contributing to virulence in 414 clinical isolates and at the same time were found to be differently regulated in the G. mellonella evolved strain are listed. Downregulated genes and upregulated genes in the evolved strain are shown

\section{Persistence of the induced virulence phenotype despite a change in the conditions that induced the phenotype}

To test whether the virulent phenotype of the evolved strain was stable, we cultivated the strain in rich LB medium over a time period of 4 days (Fig. 1b). Clearly as depicted in 
Fig. 1c, when the larvae were infected with this revertant isolate $(\mathrm{CH} 2658 \mathrm{Rev})$, the G. mellonella survival rate increased again to the level of the ancestor isolate. The revertant also exhibited the low cytotoxicity phenotype of the original clinical isolate in the A549 lung epithelial cell infection model (Supplementary Fig. S2A). We next monitored the generation time necessary for the evolved strain to revert back to the low virulence level of the ancestor isolate (Supplementary Fig. S3). Therefore, we took advantage of the finding that the evolved strain exhibited a smaller colony morphology on agar plates than compared with the original clinical CH2658 strain or the CH2658 Rev (Supplementary Fig. S4). We calculated the ratios between small and large colony morphologies, and found that after 38 generations $\sim 50 \%$ of the evolved strain reverted to the ancestor clinical isolate phenotype. Our results thus suggest a stably induced virulence phenotype that persists in the clinical isolate for several generations despite the change in the conditions that first induced this phenotype. As a control, we selected small and large colonies from the same plate after 1 and 3 days of LB passage and tested them for their G. mellonella virulence phenotype (Supplementary
Fig. S5; two small and large colonies per plate, respectively). Whereas the small colonies exhibited a virulent phenotype, large colonies showed a less virulent phenotype similar to the $\mathrm{CH} 2658$ isolate and the revertant.

\section{Phenotypic characterization of the evolved $P$. aeruginosa isolate}

We evaluated whether the evolved transcriptional phenotype of $\mathrm{CH} 2658$ IV was associated with distinct motility phenotypes and/or altered biofilm formation. Swimming and swarming were increased in the evolved strain (Fig. 4a), whereas the strain produced lower biofilm levels as determined by the use of the crystal violet assay (Fig. 4b). Replica passages of $\mathrm{CH} 2658$, which were evolved in the G. mellonella larvae in independent experiments, exhibited the same phenotypes and showed a low biofilm production that increased again to the levels of the original clinical isolate after serial passages in rich LB conditions (Supplementary Fig. S6), indicating that the CH2658 IV phenotype evolved repeatedly after passaging and was not a rare event that got picked up accidentally. We also analyzed the
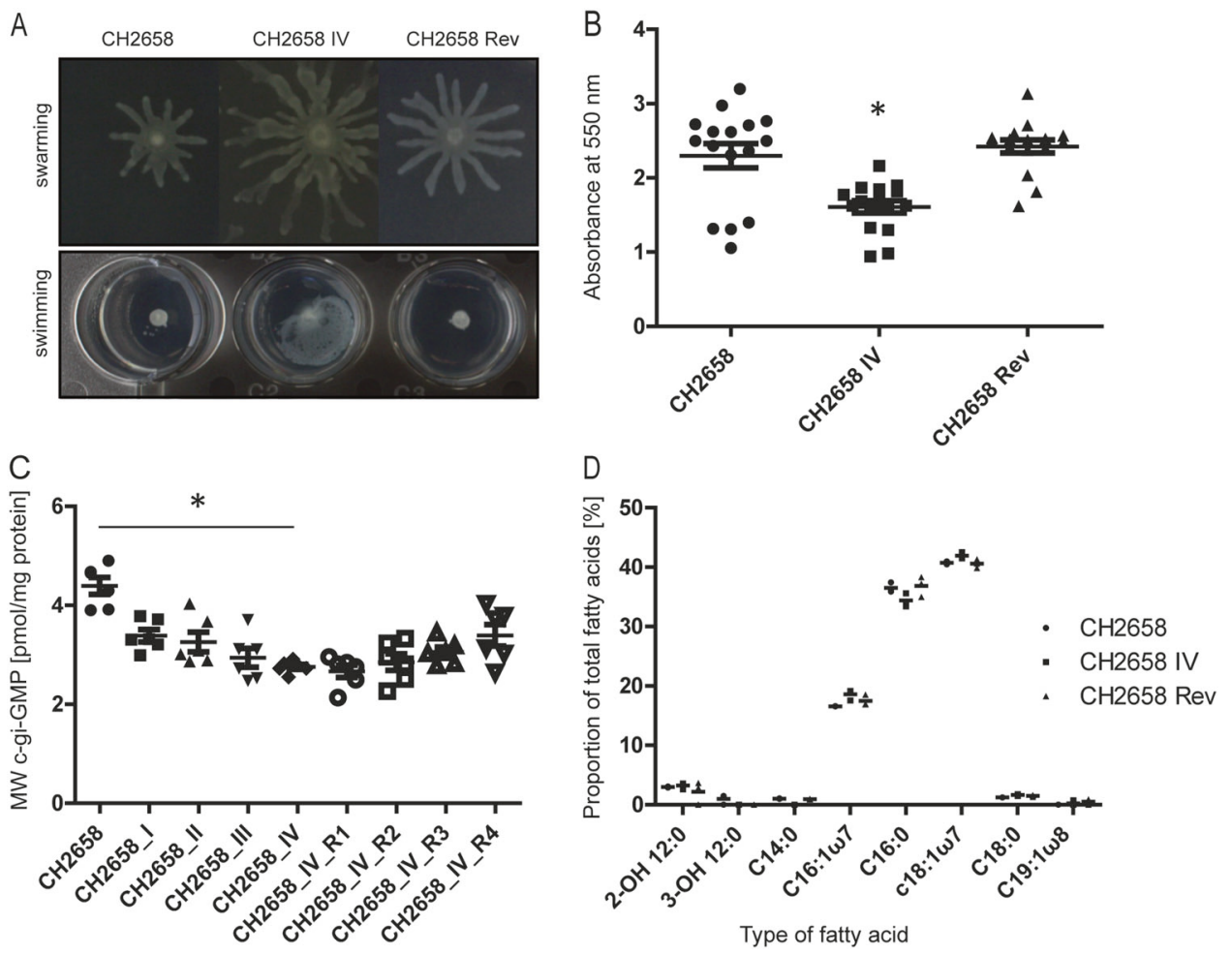

Fig. 4 Phenotypic characterization of the evolved strain. a Swarming and swimming motility of the clinical isolate (CH2658), the evolved strain (CH2658 IV), and the revertant (CH2658 Rev) was assessed following $16 \mathrm{~h}$ of incubation in BM2 medium at $30^{\circ} \mathrm{C}$ under humid conditions. One representative example of two biological replicates is shown. b Biofilm formation was analyzed using the crystal violet staining assay $(n=16)$. $\mathbf{c}$ C-di-GMP concentrations were quantified using LC-MS for all G. mellonella passages (1-4) as well as for all LB

passages (1-4). Thereby, the G. mellonella passage 4 corresponds to the evolved strain (CH2658 IV) and the LB passage 4 to the revertant (CH2658 Rev) $(n=6)$. d Fatty acid composition was measured using gas chromatography in combination with a flame ionization detector (FID) $(n=3)$. Asterisks indicate parameters that show statistically significant differences $(p<0.05)$ as determined by the Mann-Whitney $U$-test 
intracellular levels of the global second messenger c-diGMP, which is known to govern virulence, motility, and biofilm phenotypes [39]. As shown in Fig. 4c, the c-di-GMP concentration decreased with every passage in the G. mellonella larvae, whereas the c-di-GMP concentration increased again stepwise upon cultivation of the evolved strain in LB medium. We furthermore extracted fatty acids of the bacterial membrane and recorded their composition in the wild type, revertant, and the evolved strain by the use of gas chromatography in combination with a FID. However, we could not observe significant differences in the evolved strain as compared with the clinical isolate and the revertant (Fig. 4d).

To obtain more insights into the evolution of virulence in our clinical isolate, we compared RNA-Seq data of the clinical isolate (CH2658), the evolved strain (CH2658 IV), and the revertant $(\mathrm{CH} 2658 \mathrm{Rev}$, passage 4). We also included all isolates that were sampled after every single passage in the in vivo (CH2658 I-III) and in vitro conditions (CH2658 Rev1-3). As demonstrated in the PCA analysis (Fig. 5), the transcriptional profiles of the G. mellonella larvae passages I and II were quite similar and still close to the ancestor clinical isolate, whereas the profiles of passage III and IV produced distinct clusters. Strikingly, the transcription profiles of the revertants followed the corresponding in vivo passages on each step backwards.

\section{Passage of the clinical isolate in the presence of linolenic acid mimics the G. mellonella evolved phenotype}

We hypothesized that the particularly high polyunsaturated fatty acid composition in the G. mellonella larvae might serve as an environmental cue, which could be involved in the establishment of a memory virulence phenotype. We therefore evaluated whether we could evolve a virulent phenotype in the clinical isolate, if the isolate is passaged in medium supplemented with polyunsaturated fatty acids. We therefore added $5 \%$ of linolenic acid $(18: 2 \omega 6,9)$ to LB medium. Linolenic acid is a major component of the polyunsaturated fatty acid fraction in the family of lepidoptera (up to $45 \%$ of the fatty acids are linolenic acids [27]). We furthermore passaged the clinical isolate in linolenic acid-supplemented LB medium with an additional overlay of paraffin oil in order to mimic the microaerophilic environment during the infection process within the insect host. After passaging the clinical isolate eight times in the presence of linolenic acid with and without paraffin, the gene expression profile was recorded. As depicted in Supplementary Fig. S7, the global transcriptional profile of the in vivo and the in vitro linolenic acid-passaged clinical isolate clearly clustered in the PCA analysis. The genes that were commonly differentially regulated in the in vivo and the in vitro conditions are shown in Fig. 6a (and in Supplementary Table S3). Strikingly, we found an overlap of genes involved in the T3SS and glycerol metabolism. Furthermore, genes involved in biofilm formation $(a l g, p s l)$ and in starch and sucrose metabolism or fatty acid metabolism were commonly downregulated in the evolved strains as compared with the clinical isolate independent on whether they evolved in the G. mellonella larvae or in linoleic acidsupplemented medium with or without paraffin. Of note, the colony morphology did not change in the in vitro linolenic acid-passaged clinical isolate.

Interestingly, the in vitro (in the presence of linolenic acid)-evolved strains were able to kill the G. mellonella larvae to a similar degree as the in vivo-evolved strain (Fig. 6b). They were also more cytotoxic as determined in a cell culture experiment using A549 lung epithelial cells (Supplementary Fig. S2B). Furthermore, the in vitropassaged isolates produced less biofilm, even less than the in vivo-evolved strain (Fig. 6c). This was in accordance with the finding that the supplementation of linolenic acid over several generations resulted in a lower production of $\mathrm{c}$ -
Fig. 5 Transcriptional analysis uncovers phenotypic switching during passages in vivo and in vitro. PCA plot of transcriptional data of all in vivo and in vitro passages grown under rich medium conditions $(n=2)$. The clinical isolate (CH2658) and the in vivo evolved strains from passage 1-4 (CH2658_I-IV) are highlighted in blue and the in vitro passages (1-4) of the revertant (CH2658_Rev1-4) in red (circle and arrow)

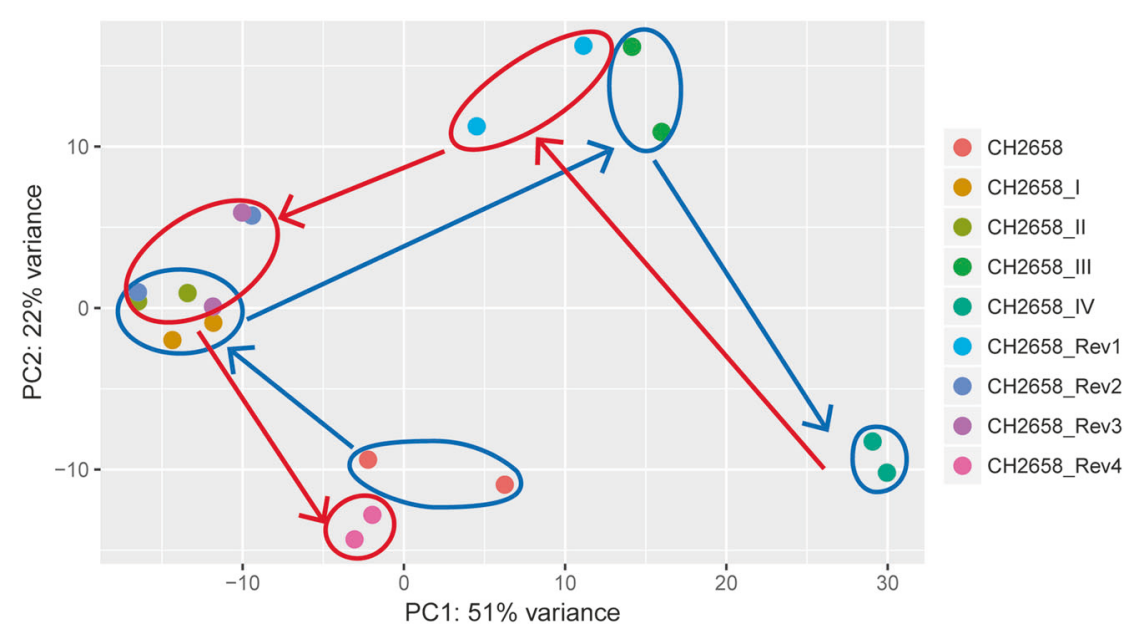



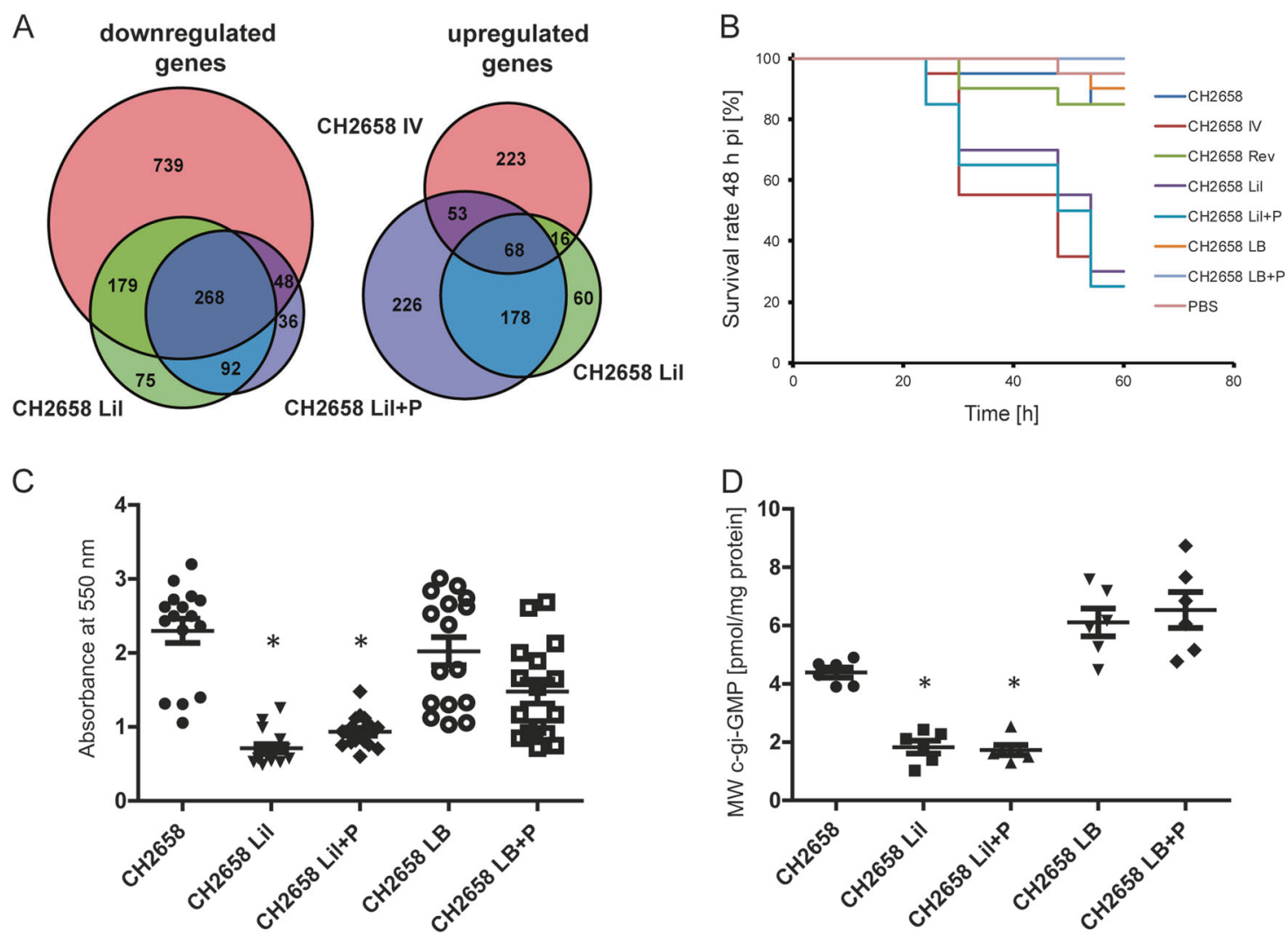

Fig. 6 Induction of a memory response upon passaging of $P$. aeruginosa in the presence of linolenic acid. a Overlapping up- or downregulated genes of the in vivo, G. mellonella passaged CH2658 IV isolate and the in vitro, LB (supplemented with linolenic acid with and without paraffin) passaged clinical isolate $\mathrm{CH} 2658$ as compared with the ancestor clinical isolate $\mathrm{CH} 2658$ are depicted in a Venn diagram (BioVenn). b Kaplan-Meier plot showing the survival rate of G. mellonella larvae infected with the clinical isolate, the evolved

strain, the revertant, and the in vitro passages over time. Mean survival rates from two biological replicates are shown. c Biofilm formation was analyzed using crystal violet staining $(n=16)$. d C-di-GMP concentrations were quantified using LC-MS $(n=6)$. Asterisks indicate parameters that show statistically significant differences $(p<0.05)$ as determined by the Mann-Whitney $U$-test. $\mathrm{CH} 2658 \mathrm{Lil}=$ clinical isolate passaged in $\mathrm{LB}$ with $5 \%$ linolenic acid, $\mathrm{CH} 2658 \mathrm{LB}=$ clinical isolate passage in $\mathrm{LB}, \mathrm{P}=$ cultures were overlayed with paraffin

di-GMP, even lower than in the in vivo-evolved strain (Fig. 6d). As a control, we passaged the clinical isolate under normal LB conditions with and without the addition of paraffin. As opposed to the passages that were cultured in the presence of linoleic acid, these passaged isolates did not become more virulent nor did they exhibit decreased biofilm forming capabilities (Fig. 6c, d).

\section{Discussion}

The broad ecological success of the opportunistic pathogen $P$. aeruginosa is based on its remarkable capability to adapt to a range of very different growth conditions. One exceedingly challenging environment is the habitat of the eukaryotic host during an infection process. This habitat provides strong selection pressures for adaptive changes. In this study we describe an adaptive mechanism that adds to the well described long-term evolutionary adaptations and short-term individual adaptations. Upon passaging in the

G. mellonella insect host, $P$. aeruginosa adopts a phenotypic response that is characterized by switching to a virulence phenotype. Remarkably, the transcriptionally reprogrammed phenotype was not linked to the acquisition of genetic variations and was sustained for several generations upon cultivation of the ex vivo virulence-adapted $P$. aeruginosa cells under rich medium conditions. The hostinduced virulence memory response could be reproduced in bacteria that were passaged in medium supplemented with excess linolenic acid, a long-chain polyunsaturated fatty acid. The G. mellonella larvae hemolymph is characterized by a high proportion of triglycerides, diacylglycerols, trehalose, and glycogen, which are released from the fat body [40-42], as well as polyunsaturated fatty acids, the majority of which are linolenic acids [27]. Our results thus indicate that the memory response of $P$. aeruginosa could be induced by exposure to a host-characteristic fatty acid composition. There are several well-described host-specific environmental factors that have an impact on and drive bacterial pathogenicity. These include the higher host 
temperature, scarcity of iron, and alterations in the concentration of phosphate, $\mathrm{pH}$, oxygen tension, and osmolality [43-46]. Furthermore, there is increasing evidence that the host-specific fatty acid composition during an infection process can be added to the list of factors that are sensed by the bacteria to induce a virulence phenotype. Host- and in vitro-derived exogenous fatty acids were demonstrated to influence bacterial membrane physiology. This resulted in altered phenotypes such as virulence, motility, antimicrobial peptide susceptibility, or biofilm formation [47-52]. It was proposed that exogenous polyunsaturated fatty acids were directly incorporated into the bacterial membrane and thus to affect membrane permeability. However, it was also argued that bacteria import exogenous fatty acids to generate acyl-CoA by $\beta$-oxidation, which can be used for endogenous phospholipid biosynthesis [53].

The memorization of cellular responses to past conditions might be important, because some of them are beneficial for future conditions. Especially in very dynamic environments, carry-over effects generate a history-dependent behavior or bacterial memory that were demonstrated to be of value [5457]. Thereby, memory responses are general strategies to transmit information between generations and can be achieved in multiple ways. In most of the instances, memory is dependent on the stability of RNAs, proteins, or on the circuit of positive feedback loops in gene-regulatory networks. However, also gene copy number variations might be responsible for carry-over effects, as the fitness effect of a gene duplication may be the result of an increase in protein dosage [58]. It was also claimed that numerous molecular steps involved in birth and decay of macromolecules (e.g., mRNA) can produce gestation and senescence periods or memory between these steps [59]. The clinical isolate of our study developed a hysteretic behavior upon passages in the infected insect host. The induced virulence phenotype was mainly characterized by high expression of genes involved in the T3SS system, but also by enhanced motility and reduced biofilm formation. Virulence, motility, and biofilm formation are well known to be governed by changes in the intracellular levels of the universal intracellular signaling molecule c-diGMP $[39,60]$. One might thus speculate that the in vivoevolved $P$. aeruginosa isolate expresses a hysteretic c-diGMP signaling behavior, which might be induced by a carryover of proteins involved in the modulation of the c-di-GMP levels, and which would be fixed even if the $P$. aeruginosa cells were gown under conditions that would not induce the observed c-di-GMP levels [61]. Future studies will have to uncover whether carry-over effects of c-di-GMP modulating proteins into the next generation result in a high- or low-level c-di-GMP and how this could be linked to the exogenous source of polyunsaturated long-chain fatty acids.

In conclusion, our study highlights the sheer diversity of mechanisms employed by bacteria to adapt expression of virulence in response to the environmental conditions such as those that prevail in the host niche. Efforts to unravel the fundamental mechanisms that drive bacterial adaptation to challenging habitats will continue to be of importance. Especially in the light of increasing antimicrobial resistance, novel anti-virulence strategies to fight acute and chronic $P$. aeruginosa infections might be further explored and developed into valuable novel therapy options [62]. Our results also imply that memory responses responsible for expression of virulence factors that compromise the host or enable the bacterial pathogen to evade host defense strategies might have been underestimated. It seems that in the future more response memories can be expected to be identified as efficient bacterial adaptation mechanisms to challenging environments. It will be an interesting task to elucidate the molecular mechanism that underlie the carryover effect to induce such memory responses, which ultimately drive survival of bacterial population in changing and challenging habitats.

Acknowledgements SH received funding by the EU (Consolidator Grant of the European Research council [724290]), by VW Vorab (Biofabrication for NIFE/VWZN3228), and the HGF (iMed, individualized Medicine). Many thanks to Agnes Nielsen, Anna-Lena Hagemann, Annette Garbe, and Esther Surges for help with sample preparation and for technical support.

\section{Compliance with ethical standards}

Conflict of interest The authors declare no competing interests.

Publisher's note: Springer Nature remains neutral with regard to jurisdictional claims in published maps and institutional affiliations.

\section{References}

1. Gellatly SL, Hancock REW. Pseudomonas aeruginosa: new insights into pathogenesis and host defenses. Pathog Dis. 2013;67:159-73.

2. Valentini M, Gonzalez D, Mavridou DAI, Filloux A. Lifestyle transitions and adaptive pathogenesis of Pseudomonas aeruginosa. Curr Opin Microbiol. 2018;41:15-20.

3. Klockgether J, Tümmler B. Recent advances in understanding Pseudomonas aeruginosa as a pathogen. F1000Res. 2017;6:1261.

4. Winstanley C, Brien SO, Brockhurst MA. Pseudomonas aeruginosa evolutionary adaptation and diversification in cystic fibrosis chronic lung infections. Trends Microbiol. 2016;24:327-37.

5. Yang L, Jelsbak L, Marvig RL, Damkiaer S, Workman CT, Rau $\mathrm{MH}$, et al. Evolutionary dynamics of bacteria in a human host environment. Proc Natl Acad Sci USA. 2011;108:7481-6.

6. Marvig RL, Sommer LM, Molin S, Johansen HK. Convergent evolution and adaptation of Pseudomonas aeruginosa within patients with cystic fibrosis. Nat Genet. 2015;47:57-64.

7. Feliziani S, Marvig RL, Luján AM, Moyano AJ, Di Rienzo JA, Krogh Johansen $\mathrm{H}$, et al. Coexistence and within-host evolution of diversified lineages of hypermutable Pseudomonas aeruginosa in long-term cystic fibrosis infections. PLoS Genet. 2014;10:e1004651.

8. Fusco G, Minelli A. Phenotypic plasticity in development and evolution: facts and concepts. Philos Trans R Soc. 2010;365:547-56. 
9. Kelly SA, Panhuis TM, Stoehr AM. Phenotypic plasticity: molecular mechanisms and adaptive significance. Compr Physiol. 2012;2:1417-39.

10. Price TD, Qvarnstro A, Irwin DE. The role of phenotypic plasticity in driving genetic evolution. Proc Biol Sci2003;270:1433-40.

11. Nicoglou A, Bradshaw A. The evolution of phenotypic plasticity: genealogy of a debate in genetics. Stud Hist Philos Biol Biomed Sci. 2015;50:67-76.

12. Lambert G, Kussell E. Memory and fitness optimization of bacteria under fluctuating environments. PLoS Genet. 2014;10: e1004556.

13. Jablonka E, Oborny B, Molnar I, Kisdi E, Hofbauer J, Czaran T. The adaptive advantage of phenotypic memory in changing environments. Philos Trans R Soc. 1995;350:133-41.

14. Casadesus J, Ari RD. Memory in bacteria and phage. Bioessays. 2002;24:512-8.

15. Wolf DM, Fontaine-bodin L, Bischofs I, Price G, Keasling J, Adam P. Memory in microbes: quantifying history-dependent behavior in a bacterium. PLoS ONE. 2008;3:e1700.

16. Norman TM, Lord ND, Paulsson J, Losick R. Memory and modularity in cell-fate decision making. Nature. 2013;503:481-6.

17. Miyata S, Casey M, Frank DW, Ausubel FM, Drenkard E. Use of the Galleria mellonella caterpillar as a model host to study the role of the type III secretion system in Pseudomonas aeruginosa pathogenesis. Infect Immun. 2003;71:2404-13.

18. Insua JL, Llobet E, Moranta D, Pérez-Gutiérrez C, Tomás A, Garmendia J, et al. Modeling Klebsiella pneumoniae pathogenesis by infection of the wax moth Galleria mellonella. Infect Immun. 2013;81:3552-65.

19. Ramarao N, Nielsen-Leroux C, Lereclus D. The insect Galleria mellonella as a powerful infection model to investigate bacterial pathogenesis. J Vis Exp. 2012;70:e4392.

20. Fuchs BB, O’Brien E, Khoury JBEl, Mylonakis E. Methods for using Galleria mellonella as a model host to study fungal pathogenesis. Virulence. 2010;1:475-82.

21. Harding CR, Schroeder GN, Collins JW, Frankel G. Use of Galleria mellonella as a model organism to study Legionella pneumophila infection. J Vis Exp. 2013;81:1-10.

22. Jander G, Rahme LG, Ausubel FM. Positive correlation between virulence of Pseudomonas aeruginosa mutants in mice and insects. J Bacteriol. 2000;182:3843-5.

23. Mak P, Zdybicka-barabas A. A different repertoire of Galleria mellonella antimicrobial peptides in larvae challenged with bacteria and fungi. Dev Comp Immunol. 2010;34:1129-36.

24. Grizanova EV, Komarov DA, Dubovskiy IM, Semenova AD, Slepneva IA, Chertkova EA. Maintenance of redox balance by antioxidants in hemolymph of the greater wax moth Galleria mellonella larvae during encapsulation response. Arch Insect Biochem Physiol. 2018;98:1-13.

25. Andrés L, Blanco A, Crispim JS, Fernandes KM, Oliveira LLDe, Pereira MF. Differential cellular immune response of Galleria mellonella to Actinobacillus pleuropneumoniae. Cell Tissue Res. 2017;370:153-68.

26. Browne N, Heelan M, Kavanagh K. An analysis of the structural and functional similarities of insect hemocytes and mammalian phagocytes. Virulence. 2013;4:597-603.

27. Rumpold BA, Schlüter OK. Nutritional composition and safety aspects of edible insects. Mol Nutr Food Res. 2013;57:802-23.

28. Hornischer K, Khaledi A, Pohl S, Schniederjans M, Pezoldt L, Casilag F, et al. BACTOME - a reference database to explore the sequence- and gene expression-variation landscape of Pseudomonas aeruginosa clinical isolates. Nucleic Acids Res. 2019;47: D716-20.

29. Lunter G, Goodson M. Stampy: a statistical algorithm for sensitive and fast mapping of Illumina sequence reads. Genome Res. 2011;21:936-9.
30. Love MI, Huber W, Anders S. Moderated estimation of fold change and dispersion for RNA-seq data with DESeq2. Genome Biol. 2014;15:1-21.

31. Hulsen T, Vlieg JDe, Alkema W. BioVenn - a web application for the comparison and visualization of biological lists using areaproportional Venn diagrams. BMC Genomics. 2008;9:1-6.

32. Robinson MD, Mccarthy DJ, Smyth GK. edgeR: a Bioconductor package for differential expression analysis of digital gene expression data. Bioinformatics. 2010;26:139-40.

33. Toole GAO. Microtiter dish biofilm formation assay. J Vis Exp. 2011;47:1-2.

34. Spangler C, Böhm A, Jenal U, Seifert R, Kaever V. A liquid chromatography-coupled tandem mass spectrometry method for quantitation of cyclic di-guanosine monophosphate. J Microbiol Methods. 2010;81:226-31.

35. Blanka A, Düvel J, Dötsch A, Klinkert B, Abraham W-R, Kaever $\mathrm{V}$, et al. Constitutive production of c-di-GMP is associated with mutations in a variant of Pseudomonas aeruginosa with altered membrane composition. Sci Signal. 2015;8:ra36.

36. Overhage J, Bains M, Brazas MD, Hancock REW. Swarming of Pseudomonas aeruginosa is a complex adaptation leading to increased production of virulence factors and antibiotic resistance. J Bacteriol. 2008;190:2671-9.

37. Abraham W-R, Hesse C. Isotope fractionations in the biosynthesis of cell components by different fungi: a basis for environmental carbon flux studies. FEMS Microbiol Ecol. 2003;46:121-8.

38. Pustelny C, Brouwer S, Müsken M, Bielecka A, Dötsch A, Nimtz $M$, et al. The peptide chain release factor methyltransferase PrmC is essential for pathogenicity and environmental adaptation of Pseudomonas aeruginosa PA14. Environ Microbiol. 2013;15:597-609.

39. Jenal U, Malone J. Mechanisms of cyclic-di-GMP signaling in bacteria. Annu Rev Genet. 2006;40:385-407.

40. Fukumura K, Konuma T, Tsukamoto Y, Nagata S. Adipokinetic hormone signaling determines dietary fatty acid preference through maintenance of hemolymph fatty acid composition in the cricket Gryllus bimaculatus. Sci Rep. 2018;8:4737.

41. Zheng H, Yang X, Xi Y. Fat body remodeling and homeostasis control in Drosophila. Life Sci. 2016;167:22-31.

42. Zhang Y, Xi Y. Fat body development and its function in energy storage and nutrient sensing in Drosophila melanogaster. J Tissue Sci Eng. 2014;6:1-8.

43. Rossi E, Paroni M, Landini P. Biofilm and motility in response to environmental and host-related signals in Gram negative opportunistic pathogens. J Appl Microbiol. 2018; https://doi.org/10. 1111/jam.14089.

44. Haddad A, Jensen V, Becker T, Häussler S. The Pho regulon influences biofilm formation and type three secretion in Pseudomonas aeruginosa. Environ Microbiol Rep. 2009;1:488-94.

45. Harrison F, Paul J, Massey RC, Buckling A. Interspecific competition and siderophore-mediated cooperation in Pseudomonas aeruginosa. ISME J. 2008;2:49-55.

46. Williams BRJ, Dehnbostel JO, Blackwell TIS. Pseudomonas aeruginosa: host defence in lung diseases. Respirology. 2010;15:1037-56.

47. Lopez MS, Tan IS, Yan D, Kang J, Mccreary M, Modrusan Z, et al. Host-derived fatty acids activate type VII secretion in Staphylococcus aureus. PNAS. 2017;114:11223-8.

48. Golubeva YA, Ellermeier JR, Cott Chubiz JE, Slauch JM. Intestinal long-chain fatty acids act as a direct signal to modulate expression of the Salmonella pathogenicity island 1 type III secretion system. mBio. 2016;7:e02170-15.

49. Norbash LV, Shults DJ, Symes SJK, Giles K. crossm Exogenous polyunsaturated fatty acids impact membrane remodeling and affect virulence phenotypes among pathogenic vibrio species. Appl Environ Microbiol. 2017;83:1-16. 
50. Liaw S, Lai H, Wang W. Modulation of swarming and virulence by fatty acids through the RsbA protein in Proteus mirabilis. Infect Immun. 2004;72:6836-45.

51. Lai H, Soo P, Wei J, Yi W, Liaw S, Horng Y, et al. The RssAB two-component signal transduction system in Serratia marcescens regulates swarming motility and cell envelope architecture in response to exogenous saturated fatty acids. J Bacteriol. 2005;187:3407-14.

52. Hobby CR, Herndon JL, Morrow CA, Peters RE, Symes SJK, Giles DK. Exogenous fatty acids alter phospholipid composition, membrane permeability, capacity for biofilm formation, and antimicrobial peptide susceptibility in Klebsiella pneumoniae. Microbiol Open. 2018; e635:1-11.

53. Yao J, Rock CO. Exogenous fatty acid metabolism in bacteria. Biochimie. 2017;141:30-9.

54. Kumar M, Joydeep G, Roy PK. How memory regulates drug resistant pathogenic bacteria? A mathematical study. Int J Appl Comput Math. 2017;3:747-73.

55. Tian T. Chemical memory reactions induced bursting dynamics in gene expression. PLoS ONE. 2013;8:e52029.

56. Mathis R, Ackermann M. Asymmetric cellular memory in bacteria exposed to antibiotics. BMC Evol Biol. 2017;17:1-14.
57. Grunert T, Monahan A, Lassnig C, Vogl C, Mu M. Deciphering host genotype-specific impacts on the metabolic fingerprint of Listeria monocytogenes by FTIR spectroscopy. PLoS ONE. 2014;9:1-12.

58. Kondrashov FA. Gene duplication as a mechanism of genomic adaptation to a changing environment. Proc Biol Sci. 2012;279:5048-57.

59. Pedraza JM, Paulsson J. Effects of molecular memory and bursting on fluctuations in gene expression. Science. 2008;319 :339-44.

60. Coggan Ka, Wolfgang MC. Global regulatory pathways and cross-talk control Pseudomonas aeruginosa environmental lifestyle and virulence phenotype. Curr Issues Mol Biol. 2012; 14:47-70.

61. Lee CK, Anda JDe, Baker AE, Bennett RR, Luo Y, Lee EY. Multigenerational memory and adaptive adhesion in early bacterial biofilm communities. PNAS. 2018;115:4471-6.

62. Starkey M, Lepine F, Maura D, Bandyopadhaya A, Lesic B, He J, et al. Identification of anti-virulence compounds that disrupt quorum-sensing regulated acute and persistent pathogenicity. PLoS Pathog. 2014;10:e1004321. 